MATHEMATICS OF COMPUTATION

Volume 74, Number 252, Pages 1953-1966

S $0025-5718(05) 01744-8$

Article electronically published on March 14, 2005

\title{
EXTENDED ADMISSIBLE FUNCTIONS AND GAUSSIAN LIMITING DISTRIBUTIONS
}

\author{
MICHAEL DRMOTA, BERNHARD GITTENBERGER, AND THOMAS KLAUSNER
}

\begin{abstract}
We consider an extension of Hayman's notion of admissibility to bivariate generating functions $f(z, u)$ that have the property that the coefficients $a_{n k}$ satisfy a central limit theorem. It is shown that these admissible functions have certain closure properties. Thus, there is a large class of functions for which it is possible to check this kind of admissibility automatically. This is realized with help of a MAPLE program that is also presented. We apply this concept to various combinatorial examples.
\end{abstract}

\section{INTRODUCTION}

When counting objects with two characteristics (e.g., size $n$ and another parameter $k: a_{n k}$ ) one usually expects that there exists the limiting distribution of the random variables $X_{n}$ defined by

$$
P\left[X_{n}=k\right]=\frac{a_{n k}}{a_{n}}
$$

(where $a_{n}=\sum_{k} a_{n k}$ ). In many cases this limiting distribution is Gaussian. More precisely, there exist sequences $\mu_{n}$ and $\sigma_{n}$ (with $\sigma_{n} \rightarrow \infty$ ) with

$$
\sum_{k \leq \mu_{n}+x \sigma_{n}} a_{n k}=a_{n} \Phi(x)+o\left(a_{n}\right)
$$

as $n \rightarrow \infty$, where

$$
\Phi(x)=\frac{1}{\sqrt{2 \pi}} \int_{-\infty}^{x} e^{-t^{2} / 2} d t .
$$

The purpose of this paper is to present a concept that allows one to decide this question directly by looking at the corresponding generating function

$$
f(z, u)=\sum_{n, k} a_{n k} z^{n} u^{k} .
$$

There are good reasons for considering generating functions. First of all, there are lots of (combinatorial) examples where the corresponding (bivariate) generating function is easy to establish. For example the coefficients of the generating function

$$
f(z, u)=e^{u\left(e^{z}-1\right)}
$$

Received by the editor August 19, 2003, and, in revised form, June 22, 2004.

2000 Mathematics Subject Classification. Primary 41A60; Secondary 68R05, 60F05, 05A16.

Key words and phrases. Hayman admissible functions, central limit theorem, automatic expansion, combinatorial enumeration.

This work has been supported by the Austrian Science Foundation FWF, grant P16053-N05. 
are the Stirling numbers of the second kind $S_{n k}$ that count the number of partitions of $k$ sets of a set of size $n$.

Second, the generating function $f(z, u)$ encodes certain characteristics of $X_{n}$ in a proper way. Expected value $\mathbf{E} X_{n}$ and variance $\operatorname{Var} X_{n}$ are given by

$$
\mathbf{E} X_{n}=\frac{\left[z^{n}\right] f_{u}(z, 1)}{\left[z^{n}\right] f(z, 1)}
$$

and

$$
\operatorname{Var} X_{n}=\frac{\left[z^{n}\right] f_{u u}(z, 1)+f_{u}(z, 1)-\left(f_{u}(z, 1)\right)^{2}}{\left[z^{n}\right] f(z, 1)},
$$

where $f_{u}$ denotes the derivative of $f$ with respect to $u$. Furthermore, the moment generating function $m_{n}(t)=\mathbf{E} e^{t X_{n}}$ is given by

$$
m_{n}(t)=\frac{\left[z^{n}\right] f\left(z, e^{t}\right)}{\left[z^{n}\right] f(z, 1)}
$$

There are numerous results on Gaussian limiting distributions related to the shape of generating functions. For instance, if the coefficients $\left[z^{n}\right] f(z, u)$ behave like a power of a function in $u$, there are results by Bender and Richmond BR83. and Gao and Richmond GR92. Extensions are due to Drmota Drm94, Gardy Gar95] (powers of functions), and Hwang [Hwa96, Hwa98] (so-called quasi-powers). Generating functions related to components of combinatorial constructions have been investigated by Flajolet and Soria [FS90, FS93].

We will examine a different approach: for example, if $f(z, 1), f_{u}(z, 1)$ and $f_{u u}(z, 1)$ are all Hayman-admissible functions (see Hay56]), for which the asymptotic expansion of the coefficients is known, then it is possible to get an asymptotic relation of $a_{n}=\left[z^{n}\right] f(z, 1)$ and consequently of $\mathbf{E} X_{n}$ and $\operatorname{Var} X_{n}$. One advantage of the class of Hayman-admissible functions is the presence of strong closure properties which are easy to test with MAPLE. The closure properties say that from a basic set of admissible functions (for which one has to verify that the conditions for Hayman-admissibility are fulfilled) one automatically gets a large class of composite functions that are Hayman-admissible as well.

There have been attempts to extend Hayman's concept: one is due to Harris and Schoenfeld [HS68] in order to get full expansions for the coefficients. Bender and Richmond [BR86, BR96] defined admissibility for functions in several variables and obtained a multidimensional normal law for the coefficients.

However, in order to prove a central limit theorem for the random variables defined above and to establish sufficiently strong closure properties for automatic treatment as well, we need a different concept. In the next section, we define the concept of extended admissibility and present the main results that can be proven for such functions. Section 3 gives a description of an implementation of our results in MAPLE. In section 4 we present some applications mainly related to set partition problems. The last section is devoted to the proofs.

\section{MAin Results}

We will now present the basic definition. The aim is to follow Hayman Hay56 as closely as possible, which will imply $f(z, 1)$ to be Hayman-admissible, and to require as little as possible for the behaviour of $f$ with respect to the second argument. This results in the following definition. 
Definition 1. A function $f(z, u)=\sum_{n, k>0} a_{n k} z^{n} u^{k}$ is called extended admissible or e-admissible if there exists $0<R \leq \infty$ such that the following conditions are satisfied:

(1) $f(z, u)$ is analytic in $\Delta_{R, \zeta}:=\{(z, u):|z|<R,|u|<1+\zeta\}$, where $\zeta>0$, and for some $R_{0}<R$ we have

$$
f(r, 1)>0, \quad R_{0}<r<R .
$$

(2) Set

$$
\begin{aligned}
& a(z, u)=z \frac{f_{z}(z, u)}{f(z, u)} \\
& b(z, u)=z a_{z}(z, u)=z \frac{f_{z}(z, u)}{f(z, u)}+z^{2} \frac{f_{z z}(z, u)}{f(z, u)}-z^{2}\left(\frac{f_{z}(z, u)}{f(z, u)}\right)^{2}, \\
& \bar{a}(z, u)=u \frac{f_{u}(z, u)}{f(z, u)}, \quad \bar{b}(z, u)=u \bar{a}_{u}(z, u), \quad c(z, u)=u a_{u}(z, u),
\end{aligned}
$$

and

$$
\varepsilon(r)=K\left(\bar{b}(r, 1)-\frac{c(r, 1)^{2}}{b(r, 1)}\right)^{-1 / 2}
$$

where $K>0$ is an arbitrary constant. Then, for each choice of $K>0$ there exists a function $\delta(r):\left(R_{0}, R\right) \rightarrow(0, \pi)$ such that for $R_{0}<r<R$ we have

$$
f\left(r e^{i \theta}, u\right) \sim f(r, u) \exp \left(i \theta a(r, u)-\frac{\theta^{2}}{2} b(r, u)\right), \quad \text { as } r \rightarrow R,
$$

uniformly for $|\theta| \leq \delta(r)$ and $u \in[1-\varepsilon(r), 1+\varepsilon(r)]$.

(3) For $R_{0}<r<R$ we have

$$
f\left(r e^{i \theta}, u\right)=o\left(\frac{f(r, u)}{\sqrt{b(r, u)}}\right), \quad \text { as } r \rightarrow R,
$$

uniformly for $\delta(r) \leq|\theta| \leq \pi$ and $u \in[1-\varepsilon(r), 1+\varepsilon(r)]$.

(4) For $r \rightarrow R$ we have $b(r, 1) \rightarrow \infty$.

(5) $b(r, u) \sim b(r, 1)$ for $r \rightarrow R$, uniformly for $u \in[1-\varepsilon(r), 1+\varepsilon(r)]$.

(6) $a(r, u)=a(r, 1)+c(r, 1)(u-1)+\mathcal{O}\left(c(r, 1)(u-1)^{2}\right)$ for $r \in\left(R_{0}, R\right)$ and $u \in[1-\varepsilon(r), 1+\varepsilon(r)]$.

(7) $\bar{a}(r, u)=\mathcal{O}(\bar{a}(r, 1))$ and $\bar{b}(r, u)=\mathcal{O}(\bar{b}(r, 1))$ for all $u$ in an arbitrary but fixed complex neighbourhood of 1 and all $r$.

(8) $\bar{b}(r, 1)-\frac{c(r, 1)^{2}}{b(r, 1)} \rightarrow \infty$ as $r \rightarrow R$.

(9) $\varepsilon(r)^{3} \bar{b}(r, 1) \rightarrow 0$ for $r \rightarrow R$.

(10) $\bar{b}(r, 1)=\mathcal{O}\left(\bar{a}(r, 1)^{2}\right)$ and $\bar{a}(r, 1)=\mathcal{O}\left(f(r, 1)^{\eta}\right)$ for every $\eta>0$.

Remark. Note that this definition implies that functions $f(z, u)$ that are e-admissible in some $\Delta_{R, \zeta}$ (in symbols $f \in \mathcal{E}_{R}$ ) are Hayman admissible (H-admissible, in symbols $\in \mathcal{H}_{R}$ ) in the first variable, i.e.,

$$
f(z, u) \in \mathcal{E}_{R} \Longrightarrow f(z, 1) \in \mathcal{H}_{R} .
$$

This follows from the fact that assumptions (10)-(5) actually define Hayman admissibility uniformly in $u$. In particular, this means that the analog of (10) for $a$ and $b$ is true by Hay56, Lemma 2]: we have

$$
b(r, 1)=o\left(a(r, 1)^{2}\right)
$$


and for every $\eta>0$, the estimates

$$
a(r, 1)=\mathcal{O}\left(f(r, 1)^{\eta}\right) \text { and } b(r, 1)=\mathcal{O}\left(f(r, 1)^{\eta}\right)
$$

hold.

Furthermore, Hayman's result applies for $f(z, 1)$ and because of uniformity in $u$ it carries over to $u \in[1-\varepsilon(r), 1+\varepsilon(r)]$. We get

Theorem 1. Let $f(z, u) \in \mathcal{E}_{R}$. Then as $r \rightarrow R$ we have

$$
\left[z^{n}\right] f(z, u)=\frac{f(r, u)}{r^{n} \sqrt{2 \pi b(r, u)}}\left(\exp \left(-\frac{(a(r, u)-n)^{2}}{2 b(r, u)}\right)+o(1)\right)
$$

uniformly in $n$ and $u \in[1-\varepsilon(r), 1+\varepsilon(r)]$.

As a consequence of this theorem we can prove

Theorem 2. Let $f(z, u) \in \mathcal{E}_{R}$ such that for sufficiently large $n$ all coefficients $a_{n k}$ are nonnegative. $X_{n}$ denotes a sequence of random variables satisfying

$$
P\left[X_{n}=k\right]=\frac{a_{n k}}{a_{n}} .
$$

Then the following central limit theorem holds:

$$
Y_{n}:=\frac{X_{n}-\bar{a}\left(r_{n}, 1\right)}{\sqrt{\left|B\left(r_{n}, 1\right)\right| / b\left(r_{n}, 1\right)}} \rightarrow \mathcal{N}(0,1)
$$

where $r_{n}$ is the positive solution of $a(r, 1)=n$ and $|B|$ is the determinant of $B$ with

$$
B(r, u)=\left(\begin{array}{ll}
b(r, u) & c(r, u) \\
c(r, u) & \bar{b}(r, u)
\end{array}\right) .
$$

Furthermore we have, as $n \rightarrow \infty$,

$$
\mathbf{E} X_{n}=\bar{a}\left(r_{n}, 1\right)+o\left(\left|B\left(r_{n}, 1\right)\right| / b\left(r_{n}, 1\right)\right)
$$

and

$$
\operatorname{Var} X_{n} \sim \frac{\left|B\left(r_{n}, 1\right)\right|}{b\left(r_{n}, 1\right)} .
$$

Note that (2.3), (2.4), and (2.5) also show that

$$
\frac{X_{n}-\mathbf{E} X_{n}}{\sqrt{\operatorname{Var} X_{n}}} \rightarrow \mathcal{N}(0,1) \text {. }
$$

Remark. We also want to mention that the proofs of Theorems 1 and 2 do not require all parts of the definition of e-admissibility. For Theorem 1 assumptions (11)-(4) are sufficient. The assertion of Theorem 2 follows from the assumptions (11) -(6), (8), and (9). However, all assumptions are needed to establish the closure properties for e-admissible functions. Those assumptions are usually satisfied for reasonably constructed functions; compare with Theorems 3 and 4 .

Theorem 3. The following two classes of functions are extended admissible:

- Let $P(z, u)$ be a polynomial in $z$ and $u$ with real coefficients written in the form $P(z, u)=\sum_{n} p_{n} z^{k_{n}} u^{l_{n}}$ by choosing an arbitrary order of the monomials. Furthermore, let $P(z, 1)=\sum_{m} b_{m} z^{m}$, i.e., $b_{m}=\sum_{n: k_{n}=m} p_{n}$. Finally, set

$$
K:=\max E \text { with } E=\left\{k_{i}+k_{j}: \operatorname{det}\left(\begin{array}{cc}
k_{i} & l_{i} \\
k_{j} & l_{j}
\end{array}\right) \neq 0\right\}
$$


and $I:=\left\{(i, j): k_{i}+k_{j}=K\right\}$. Then $e^{P(z, u)} \in \mathcal{E}_{\infty}$ if and only if the following conditions are satisfied:

(a) For every $d>1$ there exists an $m \neq \equiv 0 \bmod d$ such that $b_{m} \neq 0$. Moreover, for $m_{d}=\max \left\{m \neq \equiv \bmod d: b_{m} \neq 0\right\}$ we have $b_{m_{d}}>0$.

(b) $E$ is not empty and

$$
\sum_{(\mu, \nu) \in I} p_{\mu} p_{\nu} \operatorname{det}\left(\begin{array}{cc}
k_{\mu} & l_{\mu} \\
k_{\nu} & l_{\nu}
\end{array}\right)^{2}>0 .
$$

(c) $\max \left\{k_{j}: p_{j} \neq 0\right\}<3 K / 5$.

- If $f(z) \in \mathcal{H}_{R}, g(u)$ is analytic for $|u| \leq 1+\zeta$ and satisfies $g(1)>0$ as well as $g^{\prime}(1)+g^{\prime \prime}(1)-\frac{g^{\prime}(1)^{2}}{g(1)}>0$, then $e^{g(u) f(z)} \in \mathcal{E}_{R}$.

Remark. Note that for functions of the form $e^{P(z, u)}$ the definition of e-admissibility is too strict. In fact, there are many cases where a normal limit law occurs though the corresponding generating function is not e-admissible (compare with the examples presented in Section 4). The reason lies in condition (c), which is related to (9) of the definition. Since $P(z, u)$ are nice functions not only for $u$ close to one, this can be avoided and a multivariate normal limit law can be proved. A characterization of these cases is the topic of work in progress.

Theorem 4. Suppose that $f(z, u) \in \mathcal{E}_{R}$ and $g(z, u) \in \mathcal{E}_{R}, P(z, u)$ a polynomial with positive coefficients, and $h(z) \in \mathcal{H}_{R}$. Then the following functions are also in $\mathcal{E}_{R}$ :

- $f(z, u) \cdot g(z, u)$,

- $h(z) \cdot f(z, u)$,

- $P(z, u) \cdot f(z, u)$,

- $e^{f(z, u)}$,

- $e^{P(z, u) h(z)}$, if $P(z, u)$ is not independent of $u$,

- $e^{P(z, u)+h(z)}$, if $R=\infty$ and $P(z, u)$ is not independent of $u$,

- $f(z, u)+Q(z, u)$, where $Q(z, u)$ is an arbitrary polynomial.

Remark. For $e^{P(z, u)+h(z)}$ the positivity condition for $P(z, u)$ can be relaxed. For such a function to be e-admissible it is sufficient that $\lim _{z \rightarrow \infty} P_{u}(z, 1)+P_{u u}(z, 1)=$ $\infty$.

With help of Theorems 24 it is also possible to check automatically (with MAPLE) if a given function $f(z, u)$ is in $\mathcal{E}_{R}$, and in most cases one obtains a central limit theorem for the coefficients as well.

Theorem 2 also provides asymptotic expansions for the expected value and variance in terms of the derivatives of $f(z, u)$ (evaluated at $z=r_{n}$ and $u=1$ ). Thus, it is also of interest to obtain these asymptotic expansions automatically. For this purpose one has to solve two problems, first an asymptotic expansion for $r_{n}$ (that is, an asymptotic inversion of the function $\left.r \mapsto a(r, 1)=r f_{z}(r, 1) / f(r, 1)\right)$ and, second, an asymptotic insertion of $r_{n}$ into $\bar{a}(r, 1)$ and into $|B(r, 1)| / b(r, 1)$. For example, if $f$ is an exp-log function (that is, it is built by finitely many compositions of rational functions as well as $e^{z}$ and $\log z$ ), then we can apply the results and implementations for multiseries inversion and substitution by Salvy and Shackell SS99] (cf. also Richardson et al. RSSVdH96]) in order to compute automatically an asymptotic expression for $r_{n}$ and, consequently, for the mean and variance if 
they exist. (The problem is that not every exp-log function has an inverse that is asymptotically equal to an exp-log functionl; see Shackell [Sha93].)

\section{Description of the Program}

The implementation provides a MAPLE module named extadm with two user entry points: extadmtest and ea_addfunc. For H-admissibility tests, the equivalent function from algolib (see [Sal91]) is used.

extadmtest(fct::algebraic, var1::name, var2::name) tests if a given function is extended admissible in var1 around var $2=1$ and returns true, false, or an error if it cannot decide. It splits the given function $f c t$ in smaller parts according to its structure and uses the closure properties from Theorems 3 and 4 above.

A user can teach extadmtest about additional classes of functions. For this, the user has to provide a function impl that takes three arguments $f c t$, var1, and var2 like extadmtest itself, and returns true, false, or error depending on the e-admissibility of the tested function. This function impl is then added to extadmtest's function table by calling ea_addfunc(fname::symbol, impl). After a function fname has been added in this way, whenever it appears in a test, extadmtest calls impl(fct, var1, var2) on the subfunction.

For example: ea_addfunc('sinh', ea_sinh) would add the user-provided function ea_sinh as a decision function for any hyperbolic sine (sub-)functions.

Additional information will be printed when the variable infolevel[extadm] is set to at least 3.

\section{EXAMPLES}

In this section we present some combinatorial applications for e-admissibility. Note that many generating functions occurring in the following examples satisfy more than what is required for being e-admissible. For instance, examples 4.1 , 4.3. and 4.5 are admissible in the sense of Bender and Richmond [BR96] as well. However, there are combinatorial problems whose generating function is not BRadmissible; see example 4.4 .

4.1. Stirling numbers of the second kind. The generating function for the Stirling numbers of the second kind is $f(z, u)=e^{u\left(e^{z}-1\right)}$. Since $e^{z}-1$ is H-admissible, $f(z, u)$ is e-admissible by the second part of Theorem 3. We get

$$
a(z, u)=u z e^{z}, \quad \bar{a}(z, u)=u\left(e^{z}-1\right),
$$

and

$$
B(z, u)=\left(\begin{array}{cc}
u\left(z+z^{2}\right) e^{z} & u z e^{z} \\
u z e^{z} & u\left(e^{z}-1\right)
\end{array}\right) .
$$

The solution of $a(r, 1)=r e^{r}=n$ is $r_{n} \sim \log n-\log \log n$. Hence we get a normal limit law with asymptotic mean $\bar{a}\left(r_{n}, 1\right) \sim \frac{n}{\log n}$ and asymptotic variance

$$
\frac{\operatorname{det} B\left(r_{n}, 1\right)}{b\left(r_{n}, 1\right)}=e^{r_{n}}-1-\frac{\left(r_{n} e^{r_{n}}\right)^{2}}{\left(r_{n}^{2}+r_{n}\right) e^{r_{n}}} \sim \frac{e^{r_{n}}}{r_{n}} \sim \frac{n}{\log ^{2} n},
$$

which has already been shown by Harper Har67]. 
4.2. Permutations with bounded cycle length. Permutations with cycle length less than or equal to $\ell$ can be described by the generating function $f(z, u)=$ $e^{u e_{\ell}(z)}$, where $e_{\ell}(z)=\sum_{i=1}^{\ell} \frac{z^{i}}{i}$. Since the exponent is a polynomial, we have to check the conditions of Theorem 3 and it turns out that $f(z, u)$ is e-admissible if and only if $\ell>3$. So in this case Theorem 2 implies a central limit law. To get the asymptotic mean and variance compute

$$
\begin{aligned}
& a(z, u)=u z \frac{1-z^{\ell}}{1-z}, \quad \bar{a}(z, u)=u \sum_{i=1}^{\ell} \frac{z^{i}}{i}, \quad c(z, u)=a(z, u), \\
& b(z, u)=u z \frac{1-(\ell+1) z^{\ell}+\ell z^{\ell+1}}{(1-z)^{2}}, \quad \bar{b}(z, u)=\bar{a}(z, u) .
\end{aligned}
$$

$a(r, 1)=n$ implies $r_{n} \sim n^{1 / \ell}$ and, consequently, the asymptotic mean and variance are given by

$$
\bar{a}\left(r_{n}, 1\right) \sim \frac{n}{\ell} \quad \text { and } \quad \frac{\operatorname{det} B\left(r_{n}, 1\right)}{b\left(r_{n}, 1\right)} \sim \frac{2 \ell^{2}-2 \ell+1}{\ell^{2}(\ell-1)} n^{1-\frac{1}{\ell}}
$$

respectively.

Remark. Though for $\ell \leq 3$ the function is no longer e-admissible ( $\varepsilon$ would be too large in this case), it can be shown that the central limit law with asymptotic mean and variance as given above is true for $\ell \geq 2$. In the case $\ell=1$ the distribution degenerates. (The same holds for the first two examples in Section 4.5.)

4.3. Partitions of a set of partitions. These objects are the partition of the set of subsets of a given partition. One gets the generating function $f(z, u)=$ $e^{u\left(e^{\exp (z)-1}-1\right)}$. Again, one can compute the logarithmic derivatives and an asymptotic expression for $r_{n}$, mean and variance. This has been done by Salvy and Shackell [SS99, and they get

$$
\bar{a}\left(r_{n}, 1\right) \sim \frac{n}{\log n \log \log n} \quad \text { and } \quad \frac{\operatorname{det} B\left(r_{n}, 1\right)}{b\left(r_{n}, 1\right)} \sim \frac{n}{\log ^{2} n \log \log n} .
$$

Since $e^{e^{z}-1}-1$ is H-admissible and thus $f(z, u)$ e-admissible, Theorem 2 implies that, moreover, a central limit theorem holds.

4.4. Partitions counted by singleton blocks. When counting the number of partitions of an $n$-element set having $k$ singleton blocks, we get the generating function $f(z, u)=e^{e^{z}-1-z+z u}$. The exponent is the sum of a polynomial and $e^{z}-1-z$, an H-admissible function. Thus, by Theorem $4 f(z, u)$ is e-admissible, and therefore a central limit theorem holds. We get

$$
a(z, u)=z e^{z}-z+u z, \quad \bar{a}(z, u)=u z
$$

and

$$
B(z, 1)=\left(\begin{array}{cc}
\left(z^{2}+z\right) e^{z} & z \\
z & z
\end{array}\right) .
$$

Thus by $a(r, 1)=r e^{r}=n$ we have as before $r_{n} \sim \log n-\log \log n$, and therefore the asymptotic mean and variance are given by $\bar{a}\left(r_{n}, 1\right) \sim \log n$ and

$$
\frac{\operatorname{det} B\left(r_{n}, 1\right)}{b\left(r_{n}, 1\right)}=\frac{\left(r_{n}^{3}+r_{n}^{2}\right) e^{r_{n}}-r_{n}^{2}}{r_{n}\left(r_{n}+1\right) e^{r_{n}}} \sim r_{n} \sim \log n,
$$

respectively. 


\subsection{Other examples.}

Set partitions with bounded block size. The generating function is $\exp \left(u \sum_{i=1}^{\ell} \frac{z^{i}}{i !}\right)$, which is obviously e-admissible if and only if $\ell>3$.

Of course, different kinds of restrictions on the block size may be investigated by our method as well, since they differ from the present case only in the sum in the exponent. The same applies to restrictions of the cycle length in permutations (cf. Example 4.2).

Number of cycles of maximal allowed length in restricted permutations. Here the generating function is $\exp \left(\frac{u z^{\ell}}{\ell}+\sum_{i=1}^{\ell-1} \frac{z^{i}}{i}\right)$, which is e-admissible for $\ell>3$.

Covering complete graphs with bipartite graphs. The generating function is $\exp \left(u\left(e^{z}-1\right)^{2} / 2\right)$ (see [GJ83, BR96]), which is obviously e-admissible.

\section{Proofs}

Proof of Theorem 1, To prove this theorem Hayman's proof can be used without any change. The idea is to write the coefficient as

$$
\begin{aligned}
r^{n} \cdot\left[z^{n}\right] f(z, u) & =\frac{1}{2 \pi} \int_{-\delta}^{2 \pi-\delta} f\left(r e^{i \theta}, u\right) e^{-i n \theta} d \theta \\
& =\frac{1}{2 \pi}\left(\int_{-\delta}^{\delta}+\int_{\delta}^{2 \pi-\delta}\right) f\left(r e^{i \theta}, u\right) e^{-i n \theta} d \theta
\end{aligned}
$$

and to use the expansions (2) and (3) of the definition of admissibility to the two integrals of the right-hand side. Since (2) and (3) are uniform in $u$, the resulting expansion is uniform in $u$ as well.

Proof of Theorem 2. In order to prove the theorem, we have to show that the moment generating function of $X_{n}$,

$$
m_{n}(t)=\mathbf{E} e^{t X_{n}}=\frac{\left[z^{n}\right] f\left(r, e^{t}\right)}{\left[z^{n}\right] f(r, 1)}
$$

satisfies

$$
\mathbf{E} e^{t Y_{n}}=\exp \left(\frac{t \bar{a}(r, 1)}{\sqrt{b(r, 1)-c(r, 1)^{2} / b(r, 1)}}\right) \rightarrow e^{t^{2} / 2}
$$

for all $t \in[-K, K]$ with some $K>0$. Hay56, Lemma 4] tells us that, if $|\bar{b}(r, u)|<$ $\bar{b}(r, 1)$ for $|u-1|<2 \eta$ (which is true by (7)), then

$$
f\left(r, e^{t}\right)=f(r, 1) \exp \left(t \bar{a}(r, 1)+\frac{t^{2}}{2} \bar{b}(r, 1)+\mathcal{O}\left(\bar{b}(r, 1) t^{3}\right)\right), \quad \text { as } r \rightarrow R,
$$


uniformly for $|t|<\varepsilon(r)$. Thus by (6) we obtain (for $r=r_{n}$ )

$$
\begin{aligned}
m_{n}(t)=\exp \left(-\frac{\left(a\left(r, e^{t}\right)-a(r, 1)\right)^{2}}{2 b(r, 1)}(1+\mathcal{O}(t))\right. \\
\left.+t \bar{a}(r, 1)+\frac{t^{2}}{2} \bar{b}(r, 1)+o(1)\right)(1+\mathcal{O}(() t)) \\
=\exp \left(t \bar{a}(r, 1)+\frac{t^{2}}{2}\left(\bar{b}(r, 1)-\frac{c(r, 1)^{2}}{b(r, 1)}\right)\right. \\
\left.+\mathcal{O}\left(\frac{c(r, 1)^{2}}{b(r, 1)} t^{3}\right)+o(1)\right)(1+\mathcal{O}(() t)) .
\end{aligned}
$$

Since $c(r, 1)^{2}<b(r, 1) \bar{b}(r, 1)$ by (8) an application of (9) finally establishes the central limit theorem (2.3).

The proofs of (2.4) and (2.5) are standard. We can even show that all moments of the normalized random variable converge to the corresponding moments of the Gaussian distribution. Convergence of the moment generating function and Chernov's inequality provide exponential tail estimates for the distribution functions. Thus, it is sufficient to consider finite intervals for proving convergence of moments. However, on finite intervals monomials are bounded and, thus, (uniform) convergence of distribution functions implies convergence of bounded functionals. This completes the proof of Theorem 2 .

\section{Proof of Theorem 3 ,}

Part 1. Let $P(z, u)=\sum_{n=1}^{L} p_{n} z^{k_{n}} u^{l_{n}}$ be a polynomial satisfying the assumptions of the theorem. Since $P(r, u) \sim P(r, 1)$, uniformly in $u$, and the statement of the theorem is true for Hayman admissibility and polynomials in one variable (see Hay56, Theorem X]) satisfying condition (a), we immediately get (2) and (3) with $a(r, u)=\sum_{n} p_{n} k_{n} r^{k_{n}} u^{l_{n}}$ and $b(r, u)=\sum_{n} p_{n} k_{n}^{2} r^{k_{n}} u^{l_{n}}$ if we choose $\delta$ such that $\delta(r)=o\left(r^{-k / 3}\right)$, where $k=\max k_{n}$. Furthermore, (a) implies that the leading coefficient in $b(z, 1)$ is positive, which implies (4). Now (5) and (6) are obviously satisfied with $c(r, u)=\sum_{n} p_{n} k_{n} l_{n} r^{k_{n}} u^{l_{n}}$. Since $\bar{a}(r, u)=\sum_{n} p_{n} l_{n} r^{k_{n}} u^{l_{n}}$ and $\bar{b}(r, u)=\sum_{n} p_{n} l_{n}^{2} r^{k_{n}} u^{l_{n}}$ we immediately get (7). To show (8), as in Theorem 2 let $B$ denote the matrix of the second logarithmic derivatives of $P(z, u)$. Then by the multilinearity of the determinant we get

$$
\operatorname{det} B(r, 1)=\sum_{h=1}^{L} \sum_{i=1}^{L} p_{h} p_{i} r^{k_{h}+k_{i}}\left(\operatorname{det}\left(\begin{array}{cc}
k_{h} & l_{h} \\
k_{i} & l_{i}
\end{array}\right)\right)^{2} .
$$

Then by condition (b) the order of magnitude of $B$ is $B=\Theta\left(r^{K}\right)$ and the leading term is positive, which implies (8). From $b(r, 1)=\Theta\left(r^{k}\right)$ we obtain $\varepsilon=\Theta\left(r^{(K-k) / 2}\right)$ and by condition (c) this proves (9). Finally, note that $f$ grows exponentially while $\bar{a}$ and $\bar{b}$ do not. Moreover, $\bar{a}$ and $\bar{b}$ have the same order of magnitude and thus we have (10).

On the other hand, if the first condition is violated, then $e^{P(z, 1)}$ will not be $\mathrm{H}$-admissible and hence $e^{P(z, u)} \notin \mathcal{E}$. (8) and (9) are equivalent to (b) and (c), respectively. Therefore the three conditions in the theorem are necessary and sufficient for admissibility. 
Part 2. Let $F(z, u)=e^{g(u) f(z)}$ with $f \in \mathcal{H}_{R}$ and $g(u)$ as in the statement of the theorem. By [Hay56, Lemma 5] we have uniformly for $|\theta| \leq a(r)^{-1}$,

$$
f\left(r e^{i \theta}\right)=f(r)+i \theta r f^{\prime}(r)-\frac{\theta^{2}}{2}\left(r f^{\prime}(r)+r^{2} f^{\prime \prime}(r)\right)+\mathcal{O}\left(\theta^{3} f(r) a(r)^{3}\right) .
$$

We have

$$
\begin{array}{llrl}
a(r, u) & =r g(u) f^{\prime}(r), & \bar{a}(r, u) & =u g^{\prime}(u) f(r), \\
b(r, u) & =\left(r f^{\prime}(r)+r^{2} f^{\prime \prime}(r)\right) g(u), & & \bar{b}(r, u)=\left(u g^{\prime}(u)+u g^{\prime \prime}(u)\right) f(r), \\
c(r, u) & =r u g^{\prime}(u) f^{\prime}(r) . & &
\end{array}
$$

With these formulas the validity of (44)-(7) is easily seen. For (8) note that by Hay56, Th. III] we have

$$
f^{(k)}(r) \sim f(r)\left(\frac{a(r)}{r}\right)^{k}
$$

and thus we get

$$
\begin{aligned}
\bar{b} & -\frac{c^{2}}{b}=\left(g^{\prime}(1)+g^{\prime \prime}(1)\right) f(r)-\frac{r^{2} g^{\prime}(1)^{2} f^{\prime}(r)^{2}}{\left(r f^{\prime}(r)+r^{2} f^{\prime \prime}(r)\right) g(1)} \\
& \sim\left(g^{\prime}(1)+g^{\prime \prime}(1)-\frac{\left.g^{\prime}(1)^{2}\right)}{g(1)}\right) f(r) \rightarrow \infty .
\end{aligned}
$$

Consequently, $\varepsilon(r)^{3} \bar{b}(r, 1) \asymp f(r, 1)^{-1 / 2} \rightarrow 0$, as required in (9). As to the exponential growth of $f,(10)$ is obvious.

Finally, following Hayman [Hay56, we set $\delta(r)=f(r)^{-2 / 5}$ and (5.1) directly yields (2). By [Hay56, Lemma 6] we have

$$
\left|f\left(r e^{i \theta}\right)\right| \leq f(r)-f(r)^{1 / 7},
$$

and hence

$$
\left|F\left(r e^{i \theta}, u\right)\right| \leq F(r, u) e^{-g(u) f(r)^{1 / 7}} .
$$

Now applying Hay56, Th. III and Lemma 2] gives $B(r, u)=\mathcal{O}\left(g(u) f(r)^{1+\varepsilon}\right)$ and (3) follows.

\section{Proof of Theorem 4 ,}

Product of admissible functions. Let $f_{1}, f_{2} \in \mathcal{E}_{R}$. Clearly, the logarithmic derivatives of $f=f_{1} f_{2}$ satisfy

$$
\begin{array}{lll}
a=a_{1}+a_{2}, & b=b_{1}+b_{2}, & c=c_{1}+c_{2}, \\
\bar{a}=\bar{a}_{1}+\bar{a}_{2}, & \bar{b}=\bar{b}_{1}+\bar{b}_{2} . &
\end{array}
$$

Thus (5), (6), and (4) are fulfilled for $u \in[1-\eta, 1+\eta]$ with $\eta=\min \left(\varepsilon_{1}, \varepsilon_{2}\right)$. The validity of (77) is easy to check as well. To prove (8) observe that

$\bar{b}-\frac{c^{2}}{b} \geq \bar{b}_{1}-\frac{c_{1}^{2}}{b_{1}}+\bar{b}_{2}-\frac{c_{2}^{2}}{b_{2}} \Longleftrightarrow \frac{\left(c_{1}+c_{2}\right)^{2}}{b_{1}+b_{1}} \leq \frac{c_{1}^{2}}{b_{1}}+\frac{c_{2}^{2}}{b_{2}} \Longleftrightarrow 2 c_{1} c_{2} b_{1} b_{2} \leq c_{1}^{2} b_{2}^{2}+c_{2}^{2} b_{1}^{2}$,

which is obviously true and implies (8). Another consequence is that $\varepsilon=$ $1 / \sqrt{b-c^{2} / b} \leq \eta$; hence the domain of validity for (5), (6) , and (4) is large enough. Furthermore, this implies (9). (2) and (3) can be proven in the same way as Hay56, Th. VII], and (10) is obvious. 
Multiplication by a Hayman admissible function. If $f_{1} \in \mathcal{H}_{R}$ and $f_{2} \in \mathcal{E}_{R}$, then the same argument as in the previous section applies. Of course, here we have $c_{1}=\bar{a}_{1}=\bar{b}_{1}=0$ and $\varepsilon=\varepsilon_{2}$.

Multiplication by a polynomial. Let $f(z, u) \in \mathcal{E}_{R}$ with some positive $R \leq \infty$ and $P(z, u)$ a polynomial with positive coefficients. Due to uniformity in $u$ the proof of Hay56, Th. VIII] can be used without change to prove (2) and (3). Let $A, B, C, \bar{A}, \bar{B}$ denote the logarithmic derivatives corresponding to $P(z, u) f(z, u)$. Then we have

$$
\begin{aligned}
& A(r, u)=a(r, u)+r \frac{P_{z}(r, u)}{P(r, u)}, \\
& \bar{A}(r, u)=\bar{a}(r, u)+u \frac{P_{u}(r, u)}{P(r, u)}, \\
& B(r, u)=b(r, u)+r \frac{P_{z}(r, u)}{P(r, u)}+r^{2}\left(\frac{P_{z z}(r, u)}{P(r, u)}-\frac{P_{z}(r, u)^{2}}{P(r, u)^{2}}\right), \\
& \bar{B}(r, u)=\bar{b}(r, u)+u \frac{P_{u}(r, u)}{P(r, u)}+u^{2}\left(\frac{P_{u u}(r, u)}{P(r, u)}-\frac{P_{u}(r, u)^{2}}{P(r, u)^{2}}\right), \\
& C(r, u)=c(r, u)+r u\left(\frac{P_{z u}(r, u)}{P(r, u)}-\frac{P_{z}(r, u) P_{u}(r, u)}{P(r, u)^{2}}\right) .
\end{aligned}
$$

Since the rational terms in the equations above remain bounded when $r \rightarrow R$, contrary to $a, \bar{a}, b$, and $\bar{b}$, they do not affect the validity of (4)-(10).

Exponential of admissible functions. Let $F(z, u)=e^{f(z, u)}$. The logarithmic derivatives of $f$ are denoted as usual by $a, \bar{a}, b, \bar{b}$, and $c$, the ones of $F$ by the corresponding capital letters.

Since extended admissible functions satisfy all the conditions imposed on Hadmissible functions even uniformly in $u$, Lemmas 1-6 of Hayman Hay56 are true for extended admissible functions, too. The proofs there can be used without any change except for replacing $f(z)$ by $f(z, u)$ and derivatives by partial derivatives with respect to $z$, and the results hold uniformly in $u$. Since with the help of these lemmas Hayman proved that the exponential of an H-admissible function is $\mathrm{H}$-admissible as well, we can adopt his proof of (2) and (3). Moreover, we get

$$
B(r, 1) \rightarrow \infty \text {. }
$$

Note that

$$
\begin{aligned}
& A(r, u)=r f_{z}(r, u)=a(r, u) f(r, u), \\
& \bar{A}(r, u)=u f_{u}(r, u)=\bar{a}(r, u) f(r, u), \\
& B(z, u)=r f_{z}(r, u)+r^{2} f_{z z}(r, u)=\left(b(r, u)+a^{2}(r, u)\right) f(r, u), \\
& \bar{B}(z, u)=u f_{u}(r, u)+u^{2} f_{u u}(r, u)=\left(\bar{b}(r, u)+\bar{a}^{2}(r, u)\right) f(r, u), \\
& C(z, u)=z u f_{z u}(r, u)=(c(r, u)+a(r, u) \bar{a}(r, u)) f(r, u) .
\end{aligned}
$$

With these formulas we see that (10) is valid, since for every $\gamma, \eta>0$ we have $\bar{a} f=\mathcal{O}\left(f^{1+\gamma}\right)=\mathcal{O}\left(e^{\eta f}\right)$ and $\left(\bar{b}+\bar{a}^{2}\right) f=o\left(\bar{a}^{2} f^{2}\right)$. Moreover, we get

$$
\bar{B}-\frac{C^{2}}{B}=\left(\bar{b}+\bar{a}^{2}-\frac{(c+a \bar{a})^{2}}{b+a^{2}}\right) f \geq \frac{b \bar{b}-c^{2}}{b+a^{2}} f \rightarrow \infty
$$

where the inequality is obtained by expanding the terms, using $c=o(a \bar{a})$, which is a consequence of (8), (2.1), and (10), and performing some elementary estimates. 
The limit follows from (8) and (2.2). Furthermore, observe that since $|u-1| \leq$ $\sqrt{b+a^{2}} / \sqrt{\left(b \bar{b}-c^{2}\right) f}$ we obtain by (10) and (2.2),

$$
\begin{aligned}
|(u-1) \bar{a}| & \leq \frac{\bar{a} \sqrt{b+a^{2}}}{\sqrt{\left(b \bar{b}-c^{2}\right) f}} \rightarrow 0, \\
\left|(u-1)^{2} \bar{b}\right| & \leq \frac{\bar{b}\left(b+a^{2}\right)}{\left(b \bar{b}-c^{2}\right) f} \rightarrow 0 .
\end{aligned}
$$

Hence we immediately get (9):

$$
\varepsilon^{3} \bar{B}=\frac{\left(\bar{b}+\bar{a}^{2}\right)\left(b+a^{2}\right)^{3 / 2}}{\left(b \bar{b}-c^{2}\right)^{3 / 2} \sqrt{f}} \rightarrow 0 .
$$

Turning to (5) observe

$$
\begin{aligned}
& \frac{B(r, u)}{B(r, 1)} \\
& \quad=\frac{b(r, u)+a(r, u)^{2}}{b(r, 1)+a(r, 1)^{2}} \\
& \quad=\frac{b(r, 1)+a(r, 1)^{2}+\mathcal{O}\left(2 a(r, 1) c(r, 1)(u-1)+\left(a(r, 1) c(r, 1)+c(r, 1)^{2}\right)(u-1)^{2}\right)+o(b(r, 1))}{b(r, 1)+a(r, 1)^{2}} \\
& \quad=1+o(1),
\end{aligned}
$$

where the last equation follows from application of (10) and (2.2): we have

$$
\begin{aligned}
\frac{a c}{b+a^{2}}(u-1) & \leq \frac{a c}{\sqrt{b+a^{2}} \sqrt{\left(b \bar{b}-c^{2}\right) f}} \quad \text { by (5.5) } \\
& \leq \frac{b+\sqrt{b \bar{b}}}{a \sqrt{\left(b \bar{b}-c^{2}\right) f}} \rightarrow 0,
\end{aligned}
$$

by (10) along with $c \leq \sqrt{b \bar{b}}$ and then (10) together with (2.2), and

$$
\frac{a c+c^{2}}{b+a^{2}}(u-1)^{2} \leq \frac{a \sqrt{b \bar{b}}+b \bar{b}}{\left(b \bar{b}-c^{2}\right) f} \rightarrow 0
$$

by (5.5) together with (10) and (10) together with (2.2). This implies (5) and in conjunction with (5.2) we get (4). Next we prove (6). Set $u=e^{t}$. Then we have

$$
\begin{aligned}
\frac{A(r, u)-A(r, 1)}{C(r, 1)(u-1)} & =\frac{\frac{f(r, u)}{f(r, 1)} a(r, u)-a(r, 1)}{(c(r, 1)+a(r, 1) \bar{a}(r, 1))(u-1)} \\
& =\frac{\left(\frac{f\left(r, e^{t}\right)}{f(r, 1)}-1\right) a(r, 1)+\frac{f\left(r, e^{t}\right)}{f(r, 1)} c(r, 1) t+\mathcal{O}\left(\frac{f\left(r, e^{t}\right)}{f(r, 1)} c(r, 1) t^{2}\right)}{t(c(r, 1)+a(r, 1) \bar{a}(r, 1))} \\
& =\frac{\left(e^{t \bar{a}-t^{2} \bar{b}}-1\right) a+e^{t \bar{a}-t^{2} \bar{b}} c t+\mathcal{O}\left(e^{t \bar{a}-t^{2} \bar{b}} c t^{2}\right)}{t(c+a \bar{a})} \\
& =1+\mathcal{O}(t),
\end{aligned}
$$

where the last two lines follow from (5.7) and (5.6). Finally, (7) is obvious in view of (5.3) and (5.4). 
The class $\exp (P(z, u) h(z))$. Since e-admissible functions are closed under multiplication, it is sufficient to consider the case where $P(z, u)$ is a monomial. Then use the fact that the product of an $\mathrm{H}$-admissible function with a polynomial is again $\mathrm{H}$-admissible and combine this with the second part of Theorem 3 to get the result.

The class $\exp (P(z, u)+h(z))$. Here we have

$$
\begin{aligned}
& a(z, u)=z\left(P_{z}(z, u)+h^{\prime}(z)\right), \quad \bar{a}(z, u)=u P_{u}(z, u), \\
& b(z, u)=z\left(P_{z}(z, u)+h^{\prime}(z)\right)+z^{2}\left(P_{z z}(z, u)+h^{\prime \prime}(z)\right) \text {, } \\
& \bar{b}(z, u)=u P_{u}(z, u)+u^{2} P_{u u}(z, u), \\
& c(z, u)=z u P_{z u}(z, u)
\end{aligned}
$$

and hence

$$
\begin{aligned}
|B(z, u)| & =\left|\begin{array}{cc}
z P_{z}+z^{2} P_{z z} & z u P_{z u} \\
z u P_{z u} & u P_{u}+u^{2} P_{u u}
\end{array}\right|+\left|\begin{array}{cc}
z h^{\prime}+z^{2} h^{\prime \prime} & z u P_{z u} \\
0 & u P_{u}+u^{2} P_{u u}
\end{array}\right| \\
& =D_{1}(z, u)+D_{2}(z, u) .
\end{aligned}
$$

The growth properties of $\mathrm{H}$-admissible functions and their derivatives are well studied (see Hay56). Therefore we know the order of magnitude of $D_{1}(r, 1)$ and $D_{2}(r, 1)$ : we have $D_{2}(r, 1)=\Theta\left(r^{2} h^{\prime \prime}(r)\left(P_{u}(r, 1)+P_{u u}(r, 1)\right)\right.$ and $D_{1}=o\left(D_{2}\right)$, as $r \rightarrow \infty$. Moreover, since $\bar{b}(r, 1)$ and $c(r, 1)$ grow polynomially and $b(r, 1) \sim r^{2} h^{\prime \prime}(r)$ we get (8). The polynomial growth of $\bar{a}$ and $\bar{b}$ along with their equal order of magnitude immediately implies (10). Since $\varepsilon \sim \bar{b}^{-1 / 2}$, we have on the one hand (9) and on the other hand $\varepsilon \rightarrow 0$, which implies (2) and (4)-(7). Finally, since

$$
\exp \left(P\left(r e^{i \theta}, u\right)\right)=\mathcal{O}\left(e^{P(r, u)}\right) \quad \text { and } \quad e^{h\left(r e^{i \theta}\right)}=o\left(\frac{e^{h(r)}}{\sqrt{b(r, 1)}}\right)
$$

we get (3).

Addition of polynomials. Since every e-admissible function $f(z, u)$ grows at least exponentially, polynomials are negligibly small in comparison to $f(z, u)$ and thus $f(z, u)+P(z, u)$ is obviously e-admissible too.

Acknowledgments. The authors express their gratitude to Bruno Salvy who kindly offered access to a pilot MAPLE implementation of multiseries inversion.

\section{REFERENCES}

[BR83] Edward A. Bender and L. Bruce Richmond. Central and local limit theorems applied to asymptotic enumeration. II. Multivariate generating functions. J. Combin. Theory Ser. A, 34(3):255-265, 1983. MR0700034 (85k:05009)

[BR86] Edward A. Bender and L. Bruce Richmond. A generalisation of Canfield's formula. J. Combin. Theory Ser. A, 41(1):50-60, 1986. MR0826937 (87c:41023)

[BR96] Edward A. Bender and L. Bruce Richmond. Admissible functions and asymptotics for labelled structures by number of components. Electron. J. Combin., 3(1):Research Paper 34, approx. 23 pp. (electronic), 1996. MR1418482 (98a:05012)

[Drm94] Michael Drmota. A bivariate asymptotic expansion of coefficients of powers of generating functions. European J. Combin., 15(2):139-152, 1994. MR1261060|(94k:05014)

[FS90] Philippe Flajolet and Michèle Soria. Gaussian limiting distributions for the number of components in combinatorial structures. J. Combin. Theory Ser. A, 53(2):165182, 1990. MR.1041444 (91c:05012)

[FS93] Philippe Flajolet and Michèle Soria. General combinatorial schemas: Gaussian limit distributions and exponential tails. Discrete Math., 114(1-3):159-180, 1993. Combinatorics and algorithms (Jerusalem, 1988). MR.1217750 (94e:05021) 
[Gar95] Danièle Gardy. Some results on the asymptotic behaviour of coefficients of large powers of functions. Discrete Math., 139(1-3):189-217, 1995. Formal power series and algebraic combinatorics (Montreal, PQ, 1992). MR1336840 (96f:41038)

[GJ83] I. P. Goulden and D. M. Jackson. Combinatorial enumeration. A Wiley-Interscience Publication. John Wiley \& Sons Inc., New York, 1983. With a foreword by Gian-Carlo Rota, Wiley-Interscience Series in Discrete Mathematics. MR0702512 (84m:05002)

[GR92] Zhicheng Gao and L. Bruce Richmond. Central and local limit theorems applied to asymptotic enumeration. IV. Multivariate generating functions. J. Comput. Appl. Math., 41(1-2):177-186, 1992. Asymptotic methods in analysis and combinatorics. MR:1181718 (94b:05017)

[Har67] L. H. Harper. Stirling behavior is asymptotically normal. Ann. Math. Statist., 38:410-414, 1967. MR0211432 (35:2312)

[Hay56] W. K. Hayman. A generalisation of Stirling's formula. J. Reine Angew. Math., 196:67-95, 1956. MR0080749 (18:293f)

[HS68] Bernard Harris and Lowell Schoenfeld. Asymptotic expansions for the coefficients of analytic functions. Illinois J. Math., 12:264-277, 1968. MR.0224801 (37:400)

[Hwa96] Hsien-Kuei Hwang. Large deviations for combinatorial distributions. I. Central limit theorems. Ann. Appl. Probab., 6(1):297-319, 1996. MR1389841 (97g:60041)

[Hwa98] Hsien-Kuei Hwang. On convergence rates in the central limit theorems for combinatorial structures. European J. Combin., 19(3):329-343, 1998. MR.1621021 (99c:60014)

[RSSVdH96] Dan Richardson, Bruno Salvy, John Shackell, and Joris Van der Hoeven. Asymptotic expansions of exp-log functions. In Y. N. Lakshman, editor, ISSAC'96, pages 309-313. ACM Press, 1996. Proceedings of the 1996 International Symposium on Symbolic and Algebraic Computation. July 24-26, 1996. Zürich, Switzerland.

[Sal91] B. Salvy. Examples of automatic asymptotic expansions. SIGSAM Bulletin, 25(2):417, April 1991.

[Sha93] John Shackell. Inverses of Hardy L-functions. Bull. London Math. Soc., 25(2):150156, 1993. MR 1204067 (94a:26004)

[SS99] Bruno Salvy and John Shackell. Symbolic asymptotics: multiseries of inverse functions. J. Symbolic Comput., 27(6):543-563, 1999. MR1701094 (2000h:41039)

Department of Discrete Mathematics and Geometry, Technische Universität Wien, Wiedner Hauptstrasse 8-10/104, A-1040 Wien, Austria

E-mail address: drmota@dmg.tuwien.ac.at

Department of Discrete Mathematics and Geometry, Technische Universität Wien, Wiedner Hauptstrasse 8-10/104, A-1040 Wien, Austria

E-mail address: gittenberger@dmg.tuwien.ac.at

Department of Discrete Mathematics and Geometry, Technische Universität Wien, Wiedner Hauptstrasse 8-10/104, A-1040 Wien, Austria

E-mail address: klausner@dmg.tuwien.ac.at 